\title{
ÉTICA Y RESILIENCIA FAMILIAR PARA UNA ATENCIÓN INTEGRAL EN EL CAMPO DE LAS ENFERMEDADES RARAS
}

\author{
Martín García Parra \\ Universitat de les Illes Balears \\ m.garcia@uib.es
}

Fecha de Recepción: 27 Julio 2019

Fecha de Admisión: 25 Septiembre 2019

\section{RESUMEN}

En sociedades actuales, niños, niñas y jóvenes con enfermedades raras se enfrentan a barreras discapacitantes y excluyentes. ¿Cómo fomentar un carácter ético, resiliente y empoderado en sus familias que les permita mejorar su calidad de vida? Para responder a esta pregunta, INèDITHOS, un proyecto de Pedagogía Hospitalaria, busca mejorar la calidad de vida de niños, niñas y jóvenes con enfermedades raras a través de la intervención e investigación educativa y tecnológica desde un ámbito universitario en Mallorca, España.

En este contexto y en el marco de investigación de una tesis doctoral, pretendemos generar principios éticos interdisciplinares que sirvan como referencia a los diferentes profesionales que participan en el ámbito de la Pedagogía Hospitalaria y que dan soporte a familias con algún miembro con enfermedad rara. La metodología de investigación se está realizando en tres fases: a) revisión sistemática de la literatura científica actual sobre la relación entre ética, resiliencia familiar y empoderamiento comunitario; b) grupos focales de discusión con profesionales de los ámbitos sanitario, educativo y psico-social que se relacionen con el ámbito de las enfermedades raras para cartografiar sus perspectivas sobre una atención integral a este sector de la población; y c) análisis desde una teoría crítica social para proponer principios éticos en pedagogía hospitalaria.

Para esta comunicación presentaremos los avances en la investigación realizada, que se encuadra en el primer objetivo de la tesis doctoral. Por tanto, el resultado esperado de este ámbito es forjar un marco teórico coherente que permita cartografiar las perspectivas de los profesionales implicados en la atención integral de familias con enfermedades raras.

Palabras clave: ética; resiliencia familiar; prácticas interdisciplinarias; enfermedades raras; pedagogía hospitalaria; educación inclusiva.

\section{ABSTRACT}

Ethics and Family Resilience for Integral Care in the field of Rare Diseases. In contemporary 


\section{ÉTICA Y RESILIENCIA FAMILIAR PARA UNA ATENCIÓN INTEGRAL EN EL CAMPO DE LAS ENFERMEDADES RARAS}

societies, children and young people with rare diseases have disability barriers and social exclusion. How to build an ethical, resilient and empowered character in their families that allows them to improve their quality of life? To answer this question, INèDITHOS, a project of Hospital Pedagogy, aims to enhance their quality of life through intervention and educational and technological research from a university setting in Mallorca, Spain.

In this context and in the framework of research of a doctoral thesis, we claim to build interdisciplinary ethical principles so that they serve as reference to the different professionals that participate in the field of Hospital Pedagogy and that support families with a member with a rare disease. The methodology of the research is being carried out in three phases: a) systematic review of the current scientific literature about the relationship between ethics, family resilience and community empowerment; b) focus groups for discussion with health, education and psychosocial professionals that assist families with a member with a rare to cartograph their perspectives on integral care for this sector of the population; and c) analysis from a social critical theory to propose ethical principles in Hospital Pedagogy.

For this communication we will explain to the advances in our research, which is part of the first objective of the doctoral thesis. Therefore, the expected results of this field are to build a coherent theoretical framework to cartograph and to analyse the perspectives of professionals involved in the integral care of families with Rare Diseases.

Keywords: ethics; family resilience; interdisciplinary placement; rare diseases; hospital pedagogy; inclusive education.

\section{ANTECEDENTES DE LA TEMÁTICA}

Como preámbulo a la presente investigación, surge la inevitable pregunta: ¿Qué son las Enfermedades Raras? Siguiendo a Avellaneda (2007) "Las ER en su mayoría son enfermedades crónicas que producen una gran morbilidad y mortalidad prematura, además de un alto grado de discapacidad y dependencia y, por tanto, un deterioro significativo de la calidad de vida de los afectados" (2007, p. 179).

El constructo "raras" aparece debido a la poca prevalencia de dichas enfermedades. En este sentido, "empezó a utilizarse en el ámbito epidemiológico y sanitario en los Estados Unidos a principios de los años ochenta, cuando se acuñó el término ("Rare Diseases") para referirse a aquellas enfermedades que afectaban a menos de 200.000 personas en todo el país"(FEDER (Federación Española de Enfermedades Raras), 2018, p. 19), por tanto, no tiene ninguna connotación peyorativa en términos de discapacidad o mortalidad, ni debería tenerlo ante la sociedad.

Ante esta circunstancia, no hay una número homogéneo de prevalencia mundial. El porcentaje designado a la prevalencia de una ER dependerá del contexto socio-político. Así, según la FEDER (Federación Española de Enfermedades Raras) en el Estudio ENSERio -actualizado en 2018-, menciona que en España. y en la UE, se denominan ER aquellas enfermedades cuya prevalencia está por debajo de 5 por cada 10.000 habitantes (2018, p. 19). En la línea del estudio ENSERio (2018), las ER son un conjunto de enfermedades que mantienen un nexo fuerte con su entorno familiar y social, además, su alta diversidad interna, les hace compartir la necesidad de enfrentar, por un lado, las carencias estructurales del sistema social para atender sus demandas y, por otra, el desconocimiento y aislamiento (o dispersión) y el rechazo social.

Ante esta realidad, el proyecto INèDITHOS -nacido en el contexto universitario de las Islas Baleares- es, en palabras de sus fundadores, un proyecto de voluntariado universitario dirigido a mejorar la calidad de vida de niños y jóvenes en situación de enfermedad cuyo objetivo principal está puesto en ofrecer atención psicopedagógica a niños y jóvenes que padecen enfermedades crónicas y, de manera especial, enfermedades raras. Además, aplicando la metodología Aprendizaje-Servicio 
(ApS), INèDITHOS es un espacio de formación e investigación en pedagogía hospitalaria que pretende completar la formación universitaria de los estudiantes de la Universitat de les Illes Balears (Negre Bennasar \& Verger Gelabert, 2019).

En este campo surge la presente investigación que pertenece al proyecto "Soluciones educativas para mejorar la calidad de vida de niños con enfermedades minoritarias desde una intervención innovadora y transdisciplinar" (EDU2016-79402-R) financiado por el Ministerio de Economía, Industria y Competitividad. El proyecto manifiesta la necesidad de analizar y potenciar la interdisciplinariedad entre los profesionales educativo, sanitario y psico-social que atienden a niños y adolescentes con ER dentro del contexto de las Islas Baleares.

Siguiendo la línea de intervención e investigación, el modelo más idóneo para fomentar la calidad de vida es el centrado en la familia. Este enfoque considera que las familias son un pilar fundamental en la toma de decisiones informadas, siendo así, el centro desde donde se inicia el fomento a la calidad de vida de niños y adolescentes con ER. Por otro lado, tener un enfoque familiar permite una atención integral, que no sólo cubra las necesidades del niño o adolescente, sino también, las necesidades de la familia que además de contribuir en la salud física y emocional de sus miembros, la familia puede ser víctima potencial de los efectos de la enfermedad (Grau Rubio, 2013).

Con este enfoque de atención centrado en la familia, la interdisciplinariedad es vital para fomentar la calidad de vida, no sólo desde un ámbito educativo, sino también biomédico, psicosocial e incluso, ético y filosófico. Pero, ¿qué pilares debe seguir esta interdisciplinariedad para una correcta atención integral e inclusiva? ¿qué perspectivas tienen los profesionales implicados frente a una enfermedad rara? ¿cómo fomentar un carácter ético, resiliente y empoderado en sus familias que les permita mejorar su calidad de vida?

Estas preguntas sugieren responder desde una perspectiva reflexiva que cimiente una posición crítica ante la dificultades que se enfrenta este sector de la sociedad. El constructo teórico de normatividad vital (Canguilhem, 1991), el enfoque de empoderamiento comunitario (Rose, 2007) y el enfoque de resiliencia familiar centrado en el ciclo vital y variables clave en el sistema familiar (Rolland \& Walsh, 2006) aparecen como sustentos para una atención inclusiva, integral e interdisciplinar.

\section{OBJETIVOS DE LA INVESTIGACIÓN}

Objetivo general: Proponer un protocolo ético-pedagógico que posibilite a profesionales educativos, sanitarios y psicosociales una atención inclusiva e integral a familias con alguna enfermedad rara. Responde a la pregunta de investigación: ¿Cómo generar entornos éticos de atención integral en los sectores educativos, sanitarios y psicosociales que favorezca el afrontamiento familiar de una EERR?

Objetivo específico I (Primera fase de la tesis): Estado de la cuestión sobre la relación entre ética, resiliencia familiar y empoderamiento comunitario en el campo de las enfermedades raras. Con este objetivo se pretende responder a la siguiente pregunta de investigación: ¿Cuál es el estado de la cuestión sobre la relación entre entornos éticos profesionales, resiliencia familiar y empoderamiento comunitario en la atención sanitaria, educativa y psicológica a familias con algún miembro con ER?

\section{METODOLOGÍA Y/O INSTRUMENTOS UTILIZADOS}

Para dar respuesta al primer objetivo del plan investigación se llevó a cabo una revisión de la literatura con el objetivo de legitimar un marco teórico a través del análisis crítico de la evidencia científica sobre la temática tratada, es decir, la relación entre los constructos "resiliencia familiar", "ética" y "empoderamiento comunitario" dentro del campo de las enfermedades raras. La investi- 


\section{ÉTICA Y RESILIENCIA FAMILIAR PARA UNA ATENCIÓN INTEGRAL EN EL CAMPO DE LAS ENFERMEDADES RARAS}

gación sigue el modelo de cuatro fases para la revisión bibliográfica sistemática (Codina, 2018): Búsqueda, Evaluación, Análisis y Síntesis. En este trabajo, se presentan algunos resultados obtenidos que permiten establecer indicios de relación entre estos tres constructos teóricos.

Las bases de datos utilizadas para la primera fase de búsqueda bibliográfica de artículos son: DialnetPlus, Web os Science, SCOPUS, PubMed, EBSCOhost (seleccionando PsycINFO como fuente de información principal) y ERIC. Las palabras clave utilizadas son: Professional Ethics, Family Resilience, Community Empowerment y Rare Diseases / Chronic Diseases. Además, se utilizaron descriptores obtenidos del thesauros de DeSC y de ERIC. Así, se pretende conseguir coherencia crítica y argumentativa en los resultados esperados de la búsqueda bibliográfica.

Tabla 1. Cuadro de términos de búsqueda.

\begin{tabular}{|l|l|l|l|}
\hline \multicolumn{1}{|c|}{ Palabra clave } & Descriptor en DeSC & \multicolumn{1}{|c|}{$\begin{array}{c}\text { Thesaurus en } \\
\text { ERIC }\end{array}$} & $\begin{array}{l}\text { Sinónimos o } \\
\text { palabras cercanas de } \\
\text { interés }\end{array}$ \\
\hline -Family Resilience & -Family Resilience & $\begin{array}{l}\text {-Resilience } \\
\text { (Psychology) }\end{array}$ & $\begin{array}{l}\text {-Resilience } \\
\text {-Resilience, } \\
\text { Psychological }\end{array}$ \\
\hline -Professional Ethics & -Ethics, Professional & $\begin{array}{l}\text {-Ethics } \\
\text {-Moral Values }\end{array}$ & $\begin{array}{l}\text {-Moral } \\
\text {-Bioethics }\end{array}$ \\
\hline $\begin{array}{l}\text {-Community } \\
\text { Empowerment }\end{array}$ & $\begin{array}{l}\text {-Patient Participation } \\
\text {-Community Health } \\
\text { Planning. }\end{array}$ & $\begin{array}{l}\text {-Empowerment } \\
\text {-Citizen } \\
\text { Participation }\end{array}$ & $\begin{array}{l}\text {-Patient } \\
\text { Empowerment } \\
\text {-Patient Involvement } \\
\text {-Community } \\
\text { Involvement }\end{array}$ \\
\hline $\begin{array}{l}\text {-Rare Disease } \\
\text {-Chronic Disease }\end{array}$ & $\begin{array}{l}\text {-Rare Disease } \\
\text {-Chronic Disease }\end{array}$ & -Chronic Illness & $\begin{array}{l}\text { - Orphan Diseases } \\
\text { - Chronic Illnesses }\end{array}$ \\
\hline
\end{tabular}

Fuente: Elaboración propia.

En la fase de búsqueda se utilizaron preguntas directas que tuvieran nexo con los objetivos de la investigación. Debido a que es una investigación que tiene como foco reflexivo la teoría crítica social, se formularon las siguientes preguntas: ¿cómo se fundamenta el empoderamiento comunitario desde una visión educativa y biopsicosocial? ¿cómo se entiende la resiliencia familiar desde un enfoque socio-crítico? ¿cómo se relaciona el ethos, la resiliencia familiar y el empoderamiento comunitario desde una visión educativa y biopsicosocial en el ámbito de las enfermedades raras?

La estrategia de evaluación utilizada sigue los siguientes criterios de inclusión, exclusión y límites de la búsqueda bibliográfica:

Criterios de inclusión: artículos que tengan por objeto de estudio la relación entre ética o ethos, resiliencia familiar y empoderamiento comunitario; estudios que centren la resiliencia familiar en enfermedades crónicas y degenerativas -especialmente enfermedades raras-; estudios que fundamenten, de manera epistemológica, los constructos "resiliencia familiar", "ética" y "empoderamiento comunitario"; artículos que expongan la relación entre los constructos seleccionados y las prácticas interdisciplinares; artículos que manifiesten la perspectiva de profesionales sanitarios, educativos y psicológicos en relación con la resiliencia familiar y/o las comunidades morales de inclusión.

Criterios de exclusión: artículos donde el resumen no responda a los objetivos de la revisión bibliográfica sistemática; artículos de temáticas alejadas a las áreas de sanidad, educación, psicología y sociología; artículos que no tengan acceso a texto completo a través de las bases de datos de la biblioteca de la Universitat de les Isles Balears. 
Límites de la búsqueda bibliográfica: artículos o bibliografía preferentemente del 2010 al 2019

\section{RESULTADOS ALCANZADOS}

La fase de evaluación de la revisión bibliográfica aún está en proceso. Sin embargo, se han analizado una veintena de artículos que arrojan indicios críticos sobre la relación entre los términos ética, resiliencia familiar y empoderamiento, vistos desde una perspectiva educativa y biopsicosocial. Por tanto, los resultados obtenidos se centran en la pregunta: ¿Cómo se relaciona el ethos, la resiliencia familiar y el empoderamiento comunitario desde una visión educativa y biopsicosocial?

Los primeros indicios de la búsqueda bibliográfica indicaron la dificultad de acceder a estudios que tuvieran como eje transversal estos tres conceptos, dado que son conceptos que emanan de ámbitos totalmente dispares. Además, en el objetivo de nuestra investigación se entrecruzan modelos dispares que dificultan la relación entre los conceptos en el ámbito de una atención integral a las familias con algún miembro con enfermedad rara.

Sin embargo, hay estudios que manifiestan la necesidad de afrontar las enfermedades raras desde una nueva visión educativa y biopsicosocial (Avellaneda Fernández, Izquierdo, et al., 2007; Avellaneda Fernández, Layola, et al., 2007; FEDER (Federación Española de Enfermedades Raras), 2018; Fernández Hawrylak \& Grau Rubio, 2014; Fundació Víctor Grífols i Lucas, 2010; Izquierdo Martínez \& Avellaneda Fernández, 2003; Monzón González, Aróstegui Barandica, \& Ozerinjauregi Beldarrain, 2017; Puente-Ferreras, Barahona-Gomariz, \& Fernández-Lozano, 2011; Sauces \& Callado, 2016). Ahora bien, uno de los conflictos que se debe salvar para fomentar una atención integral e inclusiva está en establecer un nexo entre los modelos biomédicos y los modelos sociales; teniendo cuidado en no caer en radicalismos sobre cómo afrontar la realidad de las enfermedades raras.

Así, desde un modelo social, la discapacidad en su versión radical, se considera un hecho producido por el construccionismo social o institucional; mientras que un modelo médico, de corte biologicista, la discapacidad es un hecho independiente de cualquier representación simbólica (Vázquez García, 2015). Es decir, desde un enfoque radical biomédico, "el discapacitado, sea físico o cognitivo, es víctima de un accidente biológico cuyas condiciones sociales, incluidos los procesos y barreras que lo estigmatizan de forma estructural, se hacen invisibles" (Vázquez García, 2015, p. 113).

¿Cómo dilucidar una solución ante esta dicotomía? Una solución puede estar en la teoría del vitalismo racional de Georges Canguilhem, así lo proponen varios autores (Battán Horenstein, 2008; Maureira Velásquez, Tirado Serrano, \& Serrano, 2019; Nicholls, 2014; Vázquez García, 2015). El vitalismo racional de Canguilhem, filósofo francés reconocido por ser maestro de Michael Foucault, advierte que la vida es una acto de normatividad vital que es a la vez biológica y social. Para Canguilhem (1991, p. 161) el juicio acerca de lo saludable o patológico de una diferencia sólo puede establecerse a partir de la relación entre el individuo y su contexto social y vital concreto, es decir, su historia personal. Así, una formación integral de los profesionales implicados en la atención a familias con ER requiere una disposición de escucha y atención a la experiencia vivida de la familia, de respeto moral para decidir las metas vitales y que permita conocer los aspectos biológicos como sociales, educativos y culturales implicados en esa experiencia. En suma, la perspectiva híbrida, vitalista y racional de Canguilhem, entre lo biológico y lo social, incluso lo educativo y psicológico, da cabida a abordar el fomento de la resiliencia familiar y el empoderamiento comunitario.

Por un lado, la resiliencia familiar parte de una reformulación psicológica al concepto físico de resiliencia. Siguiendo a Claudia Grau (2013, p. 196) la resiliencia aplicada a la psicología, es la capacidad que tienen determinados sujetos y grupos de sobreponerse a las adversidades, de autorregenerarse de determinadas heridas y de sobreponerse a acontecimientos desestabilizadores; $y$ al conjunto de procesos sociales y personales que permiten conseguir bienestar físico y psicológico, a 
pesar de las adversidades. Por tanto, la resiliencia familiar se entiende como los procesos de interactivos que fortalecen al individuo y a la familia ante las exigencias de la enfermedad (Falicov, 1995; Masten \& Monn, 2015; McCubbin \& Patterson, 1983; Patterson, 2002; Rolland \& Walsh, 2006; Ungar, 2010; Walsh, 2004, 2016). En cuanto al contexto educativo, la resiliencia es entendida como el logro de buenos resultados educativos a pesar de la adversidad de los estudiantes (Boingboing, 2012). De esta manera, una escuela basada en la resiliencia es aquella que desarrolla prácticas educativas que ayudan a los alumnos a afrontar las dificultades de forma positiva, a tener proyectos de vida y a desarrollar sus potencialidades (Hart, Fernández Rodrigo, Molina, Izquierdo, \& Maitland, 2018).

Por otro, el concepto de empoderamiento comunitario en el ámbito sanitario se interpreta como un proceso social, educativo, sanitario, psicológico o político, mediante el cual los individuos y los grupos sociales son capaces de expresar sus necesidades, plantear sus preocupaciones, diseñar estrategias de participación en la toma de decisiones (Caldwell, Jones, Gallus, \& Henry, 2018; Wallerstein, 2006). En el ámbito de las ER, a través del empoderamiento comunitario se pretenden incrementar la calidad de vida de este sector de las sociedades (Aymé, Kole, \& Groft, 2008; European Organisation for Rare Diseases, 2005).

¿Cómo se relaciona la resiliencia familiar y el empoderamiento comunitario? Siguiendo a Walsh (2004): "Dado que la norma de la familia nuclear moderna ha dado paso a una multiplicidad de formas de organización familiar, el reto consiste en establecer políticas sociales y económicas y adoptar enfoques clínicos que avalen las nuevas realidades familiares"(2004, p. 76). Por tanto, desde una lectura biopolítica, Nikolas Rose (2007) resalta que debemos conceptualizar la economía de la biopolítica contemporánea como una economía no regida por la lógica de la mortalidad sino de la vitalidad. Es decir, un gobierno de la vida, en donde la cuestión no es tanto evitar la muerte, sino optimizar la vida. En este ideal de vitalidad, la biopolítica contemporánea consiste en una variedad de estrategias, entre ellas el empoderamiento comunitario y la resiliencia familiar, que procuran identificar, tratar, administrar o gestionar a los individuos, grupos o localidades en que el riesgo sea elevado, como es el caso de las familias con algún miembro con ER.

De esta manera, las nociones de resiliencia familiar y empoderamiento comunitario promueven un marco teórico versátil para el manejo de la conducta individual y colectiva ante las dificultades de la ER, algunas veces impredecibles, interconectadas en formas que confunden modelos lineales de causa y efecto, y donde los eventos sociales no respetan las barreras geográficas o culturales en sus implicaciones (Rose \& Lentzos, 2016).

En este baje conceptual, ¿dónde reside la ética en la relación entre la resiliencia familiar y el empoderamiento comunitario? Para responder a esta pregunta, es necesario mencionar el origen etimológico. La ética proviene de la palabra ethos que significó morada o guarida de los animales, y que más tarde, se referirá al ámbito humano, conservando el sentido de espacio vital seguro en el cual se acostumbra a habitar (Gónzalez, 1996). De esta noción, se afirma que ethos connota una forma habitual de comportamiento, hábito o costumbre. La filósofa Juliana González (1996) dirá que el ethos "es un modo habitual, continuo, de comportarse, de ser en el tiempo; forma de estabilidad y persistencia temporal" (1996, p. 10). Así, el ethos configura el carácter de un individuo o una comunidad y puede forjar la resiliencia familiar y el empoderamiento comunitario. Por tanto, es necesario fomentar una actuación ética en los profesionales ligada a constituir el ethos resiliente y empoderado para con las familias con algún miembro con ER. Además, la ética, en su carácter fundamental y pedagógico legitima actuaciones correctas de los profesionales para que salvaguarden la integridad de las familias y las personas con ER (Ayuso, Dal-Ré, \& Palau, 2016; Gaviria, 2013; Guilligan, 2013) 


\section{CONCLUSIONES}

Se considera que hay dos enfoques teóricos que posibilitan y legitiman una atención integral biopsicosocial a las familias implicadas. En este sentido: por un lado, el enfoque de resiliencia familiar (Aumann \& Hart, 2009; Boingboing, 2012; Walsh, 2016) y, por otro, un enfoque socio-crítico basado en los constructos de empoderamiento comunitario (Aymé et al., 2008; Rose, 2007; Wallerstein, 2006) y la normatividad vital que constituye un ethos o carácter para afrontar la enfermedad (Canguilhem, 1991).

Así, los conceptos de ética, resiliencia familiar y empoderamiento comunitario reafirman las posibilidades de supervivencia y crecimiento de todas las familias, y brindan un marco de referencia útil para los enfoques de la práctica profesional. "Los clínicos capaces de comprender los procesos fundamentales podrán movilizar recursos inéditos en las familias atribuladas, ayudándoles a hacer frente con mayor eficacia a las crisis de la vida y a emerger de ellas fortalecidas" (Walsh, 2002).

De esta manera la atención integral biopsicosocial a niños y adolescentes con enfermedades crónicas y/o enfermedades raras se sustenta en la interdisciplinariedad (Fernández Hawrylak \& Grau Rubio, 2014; Rosselló, Verger Gelabert, Negre Bennasar, \& Paz Lourido, 2018). Así tenemos tres campos de actuación: a) el campo educativo sustentado en la escuela inclusiva (Monzón González et al., 2017), la pedagogía hospitalaria (Negre Bennasar \& Verger Gelabert, 2017; Violant Holz, Molina Garuz, \& Pastor Vicente, 2011) y el desarrollo tecnológico que permita la inclusión educativa de los agentes implicados (Altina Cunha de Araújo, Paz-Lourido, \& Verger Gelabert, 2016; Day \& Gu, 2013); b) el campo sanitario basado en nueva lectura hacia la vida, sustentada como una normatividad vital (Battán Horenstein, 2008; Vázquez García, 2015); y c) el ámbito social desde la postura de la ethopolítica que posibilite la generación de comunidades morales de inclusión (Rose, 2007).

En conclusión, el trabajo interdisciplinar de esto tres campos, basados en la ética, la resiliencia familiar y el empoderamiento comunitario, posibilitará la deconstrucción de los discursos que enajenan las perspectivas hacia las enfermedades raras (CIBERER, n.d.), fomentar la resiliencia familiar en las familias con algún miembro con enfermedad crónica y/o enfermedad rara (Grau Rubio, 2013) y permitir la generación de comunidades morales de inclusión (Fraga, 2017).

\section{REFERENCIAS BIBLIOGRÁFICAS}

Altina Cunha de Araújo, C., Paz-Lourido, B., \& Verger Gelabert, S. (2016). Tipos de apoyo a las familias con hijos con discapacidad y su influencia en la calidad de vida familiar. Ciência \& Saúde Coletiva, 21(10), 3121-3130. https://doi.org/10.1590/1413-812320152110.18412016

Aumann, K., \& Hart, A. (2009). Helping children with complex needs bounce back. Resilient Theraphy for parents and professionals (Jessica Ki). Londres.

Avellaneda Fernández, A., Izquierdo, M., Torrent-Farnell, J., \& Ramón, J. R. (2007). Enfermedades raras: enfermedades crónicas que requieren un nuevo enfoque sociosanitario Rare Diseases: chronic diseases that need a new approach. An. Sist. Sanit. Navar, 30(2), 177-190.

Avellaneda Fernández, A., Layola, M., Izquierdo Martínez, M., Guilera, M., Badia Llach, X., \& Ramón, J. R. (2007). Impacto sociosanitario en pacientes con enfermedades raras (estudio ERES). Medicina Clinica. https://doi.org/10.1157/13112096

Aymé, S., Kole, A., \& Groft, S. (2008). Empowerment of patients: lessons from the rare diseases community. The Lancet, 371(9629), 2048-2051. https://doi.org/10.1016/S01406736(08)60875-2

Ayuso, C., Dal-Ré, R., \& Palau, F. (2016). Ética en la investigación de las Enfermedades Raras. Ergon. 
Battán Horenstein, A. (2008). Entre inocencia y conocimiento: la experiencia de la enfermedad en G. Canguilhem y M. Merleau-Ponty. A Parte Rei, (55), 1-8.

Boingboing. (2012). Resilience Framework (Children \& Young People), oct. 2012 - Adapted from Hart \& Blincow 2007. Retrieved 7 March 2019, from https://www.boingboing.org.uk/wp-content/uploads/2017/02/resilience-framework-children-and-young-people-2012-spanish-final.pdf

Caldwell, J. A., Jones, J. L., Gallus, K. L., \& Henry, C. S. (2018). Empowerment and Resilience in Families of Adults With Intellectual and Developmental Disabilities. Intellectual and Developmental Disabilities, 56(5), 374-388. https://doi.org/10.1352/1934-9556-56.5.374

Canguilhem, G. (1991). The Normal and the Pathological. New York: Zone Books.

CIBERER. (n.d.). Comunicación de Enfermedades Raras. Las asociaciones y los profesionales de la prensa. Guía práctica para adecuada presencia en medios.

Codina, L. (2018). Revisiones bibliográficas sistematizadas. Procedimientos generales y Framework para Ciencias Humanas y Sociales. Barcelona: Máster Universitario en Comunicación Social. Departamento de Comunicación. Universitat Pompeu Fabra [documento en pdf, acceso: eRepositorio UPF ].

Day, C., \& Gu, Q. (2013). Resilient teachers, resilient schools: Building and sustaining quality in testing times. Resilient Teachers, Resilient Schools: Building and Sustaining Quality in Testing Times. https://doi.org/10.4324/9780203578490

European Organisation for Rare Diseases. (2005). Rare Diseases: Understanding this public health priority. Eurodis. https://doi.org/10.1185/03007995.2011.562494

Falicov, C. J. (1995). Training to Think Culturally: A Multidimensional Comparative Framework. Family Process, 34(4), 373-388. https://doi.org/10.1111/j.1545-5300.1995.00373.x

FEDER (Federación Española de Enfermedades Raras). (2018). Estudio sobre situación de Necesidades Sociosanitarias de las personas con Enfermedades Raras en España. ESTUDIO ENSERio. Datos 2016-2017.

Fernández Hawrylak, M., \& Grau Rubio, C. (2014). Necesidades educativas, asistenciales y sociales especiales de los niños con enfermedades minoritarias: propuestas para una atención interdisciplinar. Revista Educación Inclusiva, 7(3), 97-124.

Fraga, E. (2017). Reciprocidad, identidad y reconocimiento vs. exclusión, marginalización y reificación. La ambigüedad del lazo social y las comunidades. Anacronismo e Irrupción, 7(12), 11-27.

Fundació Víctor Grífols i Lucas. (2010). La ética en los servicios de atención a las personas con discapacidad intelectual severa los servicios con discapacidad.

Gaviria, R. C. (2013). ÉTICA Y PEDAGOGÍA: una postura de reflexividad. Revista Brasileira de Educação de Jovens e Adultos, 1(1), 223-232.

Gónzalez, J. (1996). El ethos, destino del hombre (UNAM-FCE). México.

Grau Rubio, C. (2013). Fomentar la resiliencia en familias con enfermedades crónicas pedriátricas. Revista Española de Discapacidad, 1(1), 195-212.

Guilligan, C. (2013). La ética del cuidado (Fundació V, Vol. 30). Barcelona. https://doi.org/10.1109/6668.893246

Hart, A., Fernández Rodrigo, L., Molina, M. C., Izquierdo, R., \& Maitland, J. (2018). L’Enfocament de Resiliència Acadèmica en la promoció de la salut mental dels adolescents. Proposta per a la seva aplicació en centres educatius.

Izquierdo Martínez, M., \& Avellaneda Fernández, A. (2003). Enfoque interdiscipinario de las enfermedades raras: un nuevo reto para un nuevo siglo. Medicina Clinica, 121(8), 299-3003.

Masten, A. S., \& Monn, A. R. (2015). Child and Family Resilience: A Call for Integrated Science, Practice, and Professional Training. Family Relations, 64(1), 5-21. https://doi.org/10.1111/fare.12103 
Maureira Velásquez, M., Tirado Serrano, F., \& Serrano, F. T. (2019). The last lesson of Michel Foucault: a vitalism for a future philosophy. Athenea Digital. Revista de Pensamiento $e$ Investigación Social, 19(2), 2207. https://doi.org/10.5565/rev/athenea.2207

McCubbin, H. I., \& Patterson, J. M. (1983). The Family Stress Process. Marriage \& Family Review, 6(1-2), 7-37. https://doi.org/10.1300/J002v06n01_02

Monzón González, J., Aróstegui Barandica, I., \& Ozerinjauregi Beldarrain, N. (2017). Alumnado con enfermedades poco frecuentes y escuela inclusiva (Ediciones). Barcelona.

Negre Bennasar, F., \& Verger Gelabert, S. (2017). INEDITHOS: un proyecto de pedagogía hospitalaria dedicado a la mejora de la calidad de vida de niños y jóvenes con enfermedades raras a partir de la intervención e investigación con voluntariado universitario. Aula, 23(0), 107-119. https://doi.org/10.14201/aula201723107119

Negre Bennasar, F., \& Verger Gelabert, S. (2019). INÈDITHOS: INtervenció i investigació ÈDucativa I Tecnològica en pedagogia HOSpitalària. Bones Pràctiques En Entorns Pedagògics. Universitat de les Illes Balears.

Nicholls, L. (2014). Del allure en Canguilhem a la pulsión en Freud: de norma vital y social a pulsión y cultura. Ludus Vitalis, 22(42), 215-226.

Patterson, J. M. (2002). Integrating Family Resilience and Family Stress Theory. Journal of Marriage and Family, 64(2), 349-360. https://doi.org/10.1111/j.1741-3737.2002.00349.x

Puente-Ferreras, A., Barahona-Gomariz, M. J., \& Fernández-Lozano, M. P. (2011). Las enfermedades raras: naturaleza, características e intervención biopsicosocial. Portularia, 11(1), 11-23. https://doi.org/10.5218/prts.2011.0002.

Rolland, J. S., \& Walsh, F. (2006). Facilitating family resilience with childhood illness and disabilities. Current Opinion in Pediatrics, 18, 527-538.

Rose, N. (2007). The Politics of Life Itself. Biomedicie, Power, and Subjectivity in the Twenty-First Century (Princeton).

Rose, N., \& Lentzos, F. (2016). Making Us Resilient: Responsible Citizens for Uncertain Times. (S. Trnka \& C. Trundle, Eds.), Duke University Press.

Rosselló, M. R., Verger Gelabert, S., Negre Bennasar, F., \& Paz Lourido, B. (2018). Interdisciplinary care for children with rare diseases. Nursing \& Care, 5(January), 2016-2018. https://doi.org/10.15406/ncoaj.2018.05.00110

Sauces, M. O. S., \& Callado, R. R. (2016, December 1). Las enfermedades raras en España. Un enfoque social. Prisma Social. Fundacion para la Investigacion Social Avanzada.

Ungar, M. (2010). Families as navigators and negotiators: Facilitating culturally and contextually specific expressions of resilience. Family Process, 49(3), 421-435. https://doi.org/10.1111/j.15455300.2010.01331.x

Vázquez García, F. (2015). Georges Canguilhem y la biopolítica de las discapacidades. Sociología Histórica, 5, 93-126.

Violant Holz, V., Molina Garuz, M. C., \& Pastor Vicente, C. (2011). Pedagogía Hospitalaria. Bases para una atención integral (Laertes S.). Barcelona.

Wallerstein, N. (2006). What is the evidence on effectiveness of empowerment to improve health? Copenhagen.

Walsh, F. (2002). A Family Resilience Framework: Innovative Practice Applications. Family Relations, 51(2), 130-137. https://doi.org/10.1111/j.1741-3729.2002.00130.x

Walsh, F. (2004). Resiliencia Familiar: Estrategias para su fortalecimiento. Buenos Aires: Amorrortu Editores.

Walsh, F. (2016). Strengthening family resilience (3rd ed.). New York: Guilford Press. 
